\title{
Test and Book Reviews in Pediatric Neuropsychology
}

\author{
Andrew S. Davis ${ }^{1}$ \\ Received: 29 November 2017 / Accepted: 29 November 2017 / Published online: 8 December 2017 \\ (C) American Academy of Pediatric Neuropsychology 2017
}

Pediatric neuropsychology is still an emerging subspecialty, and as such, the methodology, standards of practice, and literature base are not as well established as some other areas of applied psychology. This is an exciting time for our field as we are seeing the development of new tests designed exclusively for a pediatric population with neurological and psychiatric concerns. Particularly, when viewed through the historical lens of the standardization and normative samples seen in early clinical neuropsychology, these recent tests have extensive research and development foundations, large and representative standardization samples, and outstanding psychometrics. The use of valid and reliable tests for the population being assessed is both an ethical mandate and best practice to answer diagnostic and functional referral questions.

The field of pediatric neuropsychology is also seeing a dramatic expansion in texts designed to address clinical pediatric neurodevelopmental issues. This dovetails with the continued growth in the neuroscience literature of the consideration of the role the brain-behavior relationship plays in common childhood neurological and psychiatric disorders, as well as more rare medical conditions. Practices and procedures are increasingly being codified via texts written by leading experts in our field. This is indeed a golden era in regard to the amount of published literature and assessment tools in pediatric neuropsychology.

Reviews of new tests and books by experienced practitioners and researchers benefit both novice and more seasoned clinicians by introducing them to new theories, concepts, practice approaches, and assessment measures. The Journal of Pediatric Neuropsychology welcomes reviews of new books and tests, and potential authors are encouraged to contact the Editor-in-Chief to discuss submissions. We are typically able to arrange free review copies of tests and books for the reviewer. The editorial policy is to send reviews to the publisher prior to publication to examine reviews for factual errors, although final publication decisions rest with the author of the review and Editor-in-Chief. All test and book reviews submitted to the Journal of Pediatric Neuropsychology undergo peer review. Submissions should be made electronically following the instructions for authors found on the journal homepage (http://www.springer.com/psychology/neuropsychology/ journal/40817).
Andrew S. Davis

davis@bsu.edu

Ball State University, Muncie, IN, USA 